\title{
INVERSE PROBLEM FOR NETWORKS OF LASER INTERFEROMETERS
}

\author{
PIOTR JARANOWSKI \\ Max-Planck-Society, Research Unit "Theory of Gravitation" \\ at the Friedrich-Schiller-University, 07743 Jena, Germany \\ E-mail: pio@gravi.physik.uni-jena.de \\ (Permanent address: Institute of Physics, Warsaw University Branch \\ Lipowa 41, 15-424 Biatystok, Poland \\ E-mail:pijar@cksr.ac.bialystok.pl)
}

\begin{abstract}
Estimation of the parameters of the gravitational-wave signal from a coalescing binary by a network of laser interferometers is considered. A generalization of the solution of the inverse problem found previously for the network of 3 detectors to the network of $N$ detectors is given. Maximum likelihood and least squares estimators are applied to obtain the solution. Accuracy of the estimation of the parameters is assessed from the inverse of the Fisher information matrix. The results of the Monte Carlo simulations are reported. They show that addition of the fourth detector to the network markedly improves performance of the network.

1. Introduction. The problem to estimate parameters of the gravitational-wave signal of a binary from measurements by a network of detectors has been posed by Bernard F. Schutz [1] and it was called the inverse problem. Solution of the problem enables estimation of the astrophysically interesting parameters of the binary: its distance from Earth, its position in the sky, and the chirp mass of the system. In Ref.[2] the authors have solved the problem for the network of 3 detectors using maximum likelihood and least squares estimators. In Ref.[3] the solution has been generalized to an arbitrary network of $N$ detectors. We have performed extensive Monte Carlo simulations for our proposed solution to assess the accuracy of the estimation of the binary parameters by networks of 3 and 4 detectors. The covariance matrices used to obtain the least squares estimators were calculated from the inverse of the Fisher information matrix. In this note we report on results of those simulations.
\end{abstract}

1991 Mathematics Subject Classification: 83C35, 94A13.

Research supported by KBN grant 2 P303D 02111.

The paper is in final form and no version of it will be published elsewhere. 
A solution of the special case of the inverse problem where parameters determining the location of the source in the sky are uncorrelated with other parameters has been obtained by Cutler and Flanagan [4]. Their solution was based on Bayesian estimators. They have also performed Monte Carlo simulations to assess the accuracy of estimation of the distance to the source.

2. Monte Carlo simulations. We define the network signal-to-noise ratio $d_{n}$ as $\sqrt{\sum_{I=1}^{N} d_{I}^{2}}$, where $N$ is the number of detectors and $d_{I}$ is the signal-to-noise ratio of the $I$-th detector. We have chosen two criteria to accept the signal.

1. DeteCtion CRITERION: we accept the signal if the network signal-to-noise ratio is $\geq 8.5$.

2. ESTIMATION CRITERION: we accept the signal if the distance measurement error is less than $100 \%$.

For each simulation we have generated 100000 signals uniformly distributed within a Euclidean sphere of a radius $R_{\max }$ and of randomly chosen angle parameters. The radius $R_{\max }$ was chosen in such a way that every signal with the network signal-to-noise ratio greater than 8.5 is included. To the population of the 100000 generated signals we have applied the two criteria above. We have used the spectral density of the interferometer noise (for the advanced LIGO instrumentation) taken from the paper by Finn and Chernoff [5] and we have considered only neutron star - neutron star binaries consisting of two 1.4 solar mass stars.

We have first considered the network of the 3 detectors located in the positions of the two LIGO and one VIRGO detectors and we have assumed that all detectors have the same sensitivity. For that network we have obtained (by means of additional Monte Carlo simulation) $R_{\max }=1.30 \mathrm{Gpc}$. For this value of $R_{\max } 13553$ signals met our detection criterion, out of which 2435 signals survived the estimation criterion.

In their investigation of the distance error measurements Cutler and Flanagan [4] assumed that the position of the source in the sky was known. They assumed that this was a very good approximation as the position accuracy determination was much better as compared to the distance determination. We have investigated this assumption. We have found that the number of signals that survives our two criteria when we neglect the position error increased by $\sim 30 \%$ from 2435 to 3117 (for the 3 detector LIGO/VIRGO network).

We have considered the effect of the addition of the fourth detector on the rms errors in the parameters of the binary. We assumed that the fourth detector is located in Australia near Perth and that it has the same sensitivity as the other detectors of the network. For this location we have investigated the optimal orientation of the detector for the estimation of the distance to the binary (and not for estimation of the other parameters). For this purpose we have generated 100000 signals within the Euclidean sphere of the radius of $1 \mathrm{Gpc}$. To the generated signals we have applied our two criteria. We have obtained that the number of signals meeting the detection criterion depends very weakly on the orientation angle (this number deviates from its maximum value by less than 
$1 \%)$. However the number of events surviving both criteria depends significantly on the orientation angle. It deviates from its maximum value at the orientation angle of $86^{\circ}$ by more than $20 \%$.

In simulations with 4 detectors we have assumed the optimum orientation angle of $86^{\circ}$. By means of additional Monte Carlo simulation we have found that the $R_{\max }=$ $1.56 \mathrm{Gpc}$ and we again generated 100000 signals in the Euclidean sphere of this radius. Now 12123 signals met the detection criterion, out of which 3066 signals survived the estimation criterion. Thus adding a fourth detector to the network increased the fraction of detectable events that survived the estimation criterion from $\sim 18 \%$ to $\sim 25 \%$.

For the network signal-to-noise ratio $d_{n}=16$ the simulations have shown the following improvements in the accuracy when going from the network of 3 detectors to the network of 4 detectors. The distance parameter $R$ of the average signal that met the two criteria above increased from $360 \mathrm{Mpc}$ to $440 \mathrm{Mpc}$. For these distances the relative rms errors $\Delta R / R$ in the distance and the chirp mass $\Delta \mathcal{M} / \mathcal{M}$ for the two networks were almost the same and they were equal to around $50 \%$ and $15 \%$, respectively. The rms error $\Delta \Omega$ in the position of the source has significantly decreased from $6.4 \times 10^{-3}$ sr to $1.8 \times 10^{-3} \mathrm{sr}$.

\section{References}

[1] B.F. Schutz, Determining the Hubble constant from gravitational wave observations, Nature 323 (1986), 310.

[2] P. Jaranowski and A. Królak, Optimal solution to the inverse problem for the gravitational wave signal of a coalescing compact binary, Phys. Rev. D 49 (1994), 1723.

[3] P. Jaranowski, K. D. Kokkotas, A. Królak and G. Tsegas, On estimation of parameters of the gravitational-wave signal from a coalescing binary by a network of detectors, Clas. Quantum Grav. 13 (1996), 1279.

[4] C. Cutler and E. E. Flanagan, Gravitational waves from merging compact binaries: How accurately can one extract the binary's parameters from inspiral waveform, Phys. Rev. D 49 (1994), 2658.

[5] L. S. Finn and D. F. Chernoff, Observing inspiral in gravitational radiation: One interferometer, Phys. Rev. D 47 (1993), 219. 